After comments and discussion by Messrs Jackson, Jones, Slocum, van Maanen, Gregory apd Verbaandert, two resolutions were adopted.

The first was proposed by Dr Jackson.

"Commission 34 recommends that no programme for the determination of solar parallax should be undertaken at the opposition of Eros in I937, but urges observatories in the northern hemisphere to obtain positions of Eros with instruments comparable in focal length to the astrographic telescopes, and with meridian circles, over as long an arc as possible."

The second resolution was proposed by Prof. Stroobant.

"Commission 34 would welcome a grant of $£$ Ioo for its work during the next three years, to be used to assist Dr Witt in his investigation of the orbit of Eros."

\title{
COMMISSION 35. (STELLAR CONSTITUTION.)
}

\author{
President: Sir ARThur EDdington. \\ SECRETARY: Prof. H. N. RusSELl.
}

The Commission spent an hour in a general discussion of problems of stellar constitution. The report as printed was unanimously adopted.

\section{COMMISSION 36. (SPECTROPHOTOMETRY.)}

President: Prof. H. H. Plaskett.

SECRETARY: Dr R. O. REDMAN.

In view of the wide range of topics covered by the Commission, it was agreed that it would be desirable to press for the nomination of a new president for the Commission each three years.

\section{Report}

In a discussion of stellar "temperatures" (§ 2.I) Prof. Russell referred to the importance of "excitation temperatures", as determined from the ratio of numbers of atoms in two states of different energy in the atom. Dr Minnaert pointed out that this was essentially only a "brightness temperature" to which Prof. Russell agreed, but added that in this way "brightness temperatures" could be found for those stars for which no measures of angular diameter were available. Dr Kienle and Mr Greaves jointly made the following suggestions on "stellar temperature" designations:

I. The designation "effective temperature" should no longer be used for temperatures which are really only "colour temperatures". The symbol $T_{e}$ should be reserved for true effective temperatures, defined as the temperature of a full radiator ("black body") which emits the same amount of total radiation as the star.

2. All that can be done by spectrophotometric observations of the continuous spectrum of the stars is to derive relative monochromatic magnitudes. Observers should therefore be requested to publish their observations either in the form of relative monochromatic magnitudes $\Delta m_{\lambda}=m_{\lambda}{ }^{*}-m_{\lambda}{ }^{0}$,

or relative gradients $G=0.92 \mathrm{I} \frac{d \Delta m_{\lambda}}{d \mathrm{I} / \lambda}$, 
referred to one, or to the mean of a few standard B and A type stars (characterized by the index zero).

3. "Gradient temperatures", $T_{g}$, may be derived from relative gradients according to the relations

$$
\begin{aligned}
& G=\Phi-\Phi_{0}, \\
& \Phi=\frac{c_{2}}{T_{g}}\left(\mathrm{I}-e^{-c_{2} / \lambda T_{g}}\right)^{-1} .
\end{aligned}
$$

When referring to such "gradient temperatures" care should be taken to mention the wave-length interval from which the gradients have been derived, since the relation between "gradient temperature" and "effective temperature" varies with the wave-length.

Dr Shapley drew attention to the importance of Dr Carroll's work on uniformity of sensitivity over the photographic plate $(\$ 3.33)$, and pointed out that if uniformly sensitive plates could be obtained commercially not only spectrophotometry, but also stellar photometry, would be revolutionized. Dr Carroll described some of the results of his work, and after further discussion the following resolution was proposed for adoption by the General Assembly:

"The International Astronomical Union at its fifth meeting in Paris wishes to draw attention to the fundamental importance in photometric and spectrophotometric problems of the production of photographic plates with more nearly uniform sensitivity over the surface, and expresses the desire that the research laboratories of the various plate manufacturers be encouraged to investigate a problem which vies in importance with that of increasing plate sensitivity."

Dr Carroll emphasized the desirability of determining the transmission of a number of standard filters, and circulating these filters among institutions desirous of testing their methods of calibration (\$3.34). It was generally agreed that this was a desirable proposal, and Dr Minnaert offered to make the Heliophysical Institute at Utrecht a centre for the intercomparison of transmissions determined at several standardizing laboratories, and to loan such filters on request to observatories engaged in spectrophotometry.

The draft report was then adopted with one textual modification.

\section{Symposium}

A general discussion on methods of calibration and standardization in photographic spectrophotometry was then opened by Dr Minnaert, Dr Chalonge, Mr Greaves and Dr Kienle. These four opening talks in fairly complete detail will be found as an appendix to the report of the Commission. Following these talks discussion became general.

With reference to methods of calibration Dr Ohman referred to a graphical method of constructing characteristic curves for prism crossed by grating spectra, and described in further detail his method of getting constant $\Delta \log I$ by placing an Iceland spar crystal back of the slit (Report, \$3.34). Dr Kienle described and gave the results of the calibration of a wire grating, (i) by measuring the grating space, (ii) by absolute measures of zero and first order images, and (iii) by a comparison of images taken with and without the grating. In place of the "half aperture" used at Greenwich, he recommended a Kreuzblende, as first applied many years ago by Stark. Mr Greaves then described how the half-aperture method was used at 
Greenwich, first in the calibration spectrograph to check the transmission of the step slit, and secondly in the stellar spectrograph as a check against differences of conditions in the laboratory and dome. He agreed that with an unwidened spectrum an ordinary half aperture would alter the character of the image, but he did not believe that this effect would be important with the broadened spectra used at Greenwich. Dr Minnaert pointed out that possible difficulties in image formation with both the half aperture and the Kreuzblende would be avoided by a slow rotation of the diaphragm.

With regard to standards of energy distribution Dr Kienle asked why $\mathrm{Dr}$ Minnaert did not use a laboratory black body as his ultimate standard of energy distribution. In reply Dr Minnaert pointed out that it is the energy distribution in some standard in which we are interested, and the more directly this energy distribution is determined the better. Published investigations and actual experience show that it is difficult to build and satisfactorily to operate a laboratory black body. Dr Minnaert then gave further details of the method of primary standardization used at Utrecht, and referred to the necessary preliminary tests for reflectivity of the thermocouple or bolometer as a function of wave-length, as well as the simple test for linearity of thermocouple and galvanometer. With regard to secondary standardization further details were given of the new methods developed at Utrecht for the determination of the emissivity of tungsten. Mr Greaves and Dr Kienle referred to difficulties which have arisen from the use of the emissivities of tungsten as a means of standardization, but Dr Minnaert did not believe that these difficulties would be serious provided tungsten filaments free from thorium impurities were used. If such impurities were believed to be present then a primary standardization, which depended in no way upon the emissivity of tungsten, should be used. Dr Carroll stated that in his opinion the absolute measures, as described by Dr Minnaert, should be regarded as the final standard; if a laboratory black body is used, the only rigorous test of its "blackness" lies in an absolute determination of its energy distribution.

With reference to stellar standards of energy distribution Dr Barbier gave an admirable review of the work done on this subject to the present. Dr Kienle and $\mathrm{Mr}$ Greaves opposed setting up at the present any definite scale of stellar "temperatures". 